\title{
Intermittent docetaxel chemotherapy is feasible for castration-resistant prostate cancer
}

\author{
HARUKI KUME, TAKETO KAWAI, MASAYOSHI NAGATA, TAKESHI AZUMA, HIDEYO MIYAZAKI, \\ MOTOFUMI SUZUKI, TETSUYA FUJIMURA, TOHRU NAKAGAWA, HIROSHI FUKUHARA and YUKIO HOMMA
}

Department of Urology, The University of Tokyo Hospital, Tokyo 113-8655, Japan

Received October 1, 2014; Accepted November 14, 2014

DOI: $10.3892 / \operatorname{mco} .2014 .469$

\begin{abstract}
This study was conducted with the aim to investigate the feasibility of intermittent treatment with docetaxel chemotherapy for castration-resistant prostate cancer (CRPC). A total of 51 men with CRPC received docetaxel at $75 \mathrm{mg} / \mathrm{m}^{2}$ every 3 weeks combined with oral dexamethasone 1.0-2.0 mg/day between 2008 and 2013. The prostate-specific antigen (PSA) level was monitored every 3 weeks. Chemotherapy was suspended when the serum PSA level decreased to $<4 \mathrm{ng} / \mathrm{ml}$, with a reduction rate of $>50 \%$ from the baseline. Treatment was resumed when serum PSA increased to $>2 \mathrm{ng} / \mathrm{ml}$, with an increase rate of $>50 \%$ from the nadir. Of the 51 cases, 27 (52.9\%) qualified for intermittent treatment; 17 patients received two courses of docetaxel chemotherapy and 10 received three courses. The median off-treatment interval was 266 days for the first drug holiday, 129.5 days for the second and 146.5 days for the third. The multivariate analysis indicated low baseline PSA (<median, $30.55 \mathrm{ng} / \mathrm{ml}$; odds ratio $=0.059, \mathrm{P}=0.010)$ and low Gleason score at diagnosis $(\leq 7$; odds ratio $=0.016, \mathrm{P}=0.040)$ as significant factors for receiving intermittent therapy. The overall survival was better in intermittent cases (hazard ratio $=2.98$ by log-rank test, $\mathrm{P}=0.023$ ). During the off-treatment period, leukopenia, thrombopenia, appetite loss, diarrhea, alopecia, nail changes and fatigue subsided (0.0-11.1\%), whereas sensory and/or motor neuropathy persisted in 12 of the 27 cases (44.4\%). Therefore, our intermittent regimen of docetaxel chemotherapy was found to be feasible for CRPC patients, since it may reduce adverse events without compromising the oncological outcome.
\end{abstract}

Correspondence to: Dr Haruki Kume, Department of Urology, The University of Tokyo Hospital, 7-3-1 Hongo, Bunkyo-ku, Tokyo 113-8655, Japan

E-mail: Kumeh-uro@h.u-tokyo.ac.jp

Key words: intermittent therapy, docetaxel, castration-resistant prostate cancer

\section{Introduction}

The treatment for advanced prostate cancer is initially surgical or medical castration, with or without concomitant use of antiandrogens. Once the cancer becomes refractory to these treatments or becomes castration-resistant prostate cancer (CRPC), second-line treatments, such as chemotherapy, immunotherapy and molecular-targeted therapy, may be indicated. The National Comprehensive Cancer Network and European Association of Urology guidelines recommend docetaxel, sipuleucel-T and novel hormonal drugs targeting endocrine pathways, including abiraterone acetate and enzalutamide $(1,2)$. Docetaxel has been one of the standard chemotherapy treatments for CRPC since 2004, when the TAX 327 and SWOG 99-16 studies demonstrated the superiority of docetaxel-based chemotherapy over mitoxantrone plus prednisone $(3,4)$.

However, docetaxel has been associated with multiple adverse events; grade 3 or 4 neutropenia occurred in $32 \%$ of the cases in the TAX 327 study and $93.7 \%$ of the cases in a Japanese phase II study (5). Alopecia and fatigue are the most common non-hematological adverse effects noted in $~ 50-70 \%$ of the cases. Nausea, vomiting, diarrhea, nail changes and neuropathy are also common, occurring in $\sim 30-40 \%$ of the cases (3).

Intermittent treatment is one of the measures applied to reduce these adverse events; intermittent or suspended treatment may provide the patients with 'drug holidays', which may also serve as a monitoring phase of therapeutic responses. However, a limited number of studies have investigated intermittent docetaxel treatment and no practical intermittency policy has been reported (6-8). In the present study, we aimed to establish a regimen for intermittent docetaxel treatment and prospectively evaluate its feasibility.

\section{Patients and methods}

Patient recruitment. This study was approved by the Institutional Ethics Committee of our hospital (no. 3124). Patients who were scheduled to receive docetaxel chemotherapy for CRPC were recruited between January, 2008 and October, 2012. The CRPC status was defined as $>25 \%$ increase of the serum prostate-specific antigen (PSA) level with values $\geq 2.0 \mathrm{ng} / \mathrm{ml}$ obtained on two consecutive measurements taken at least 


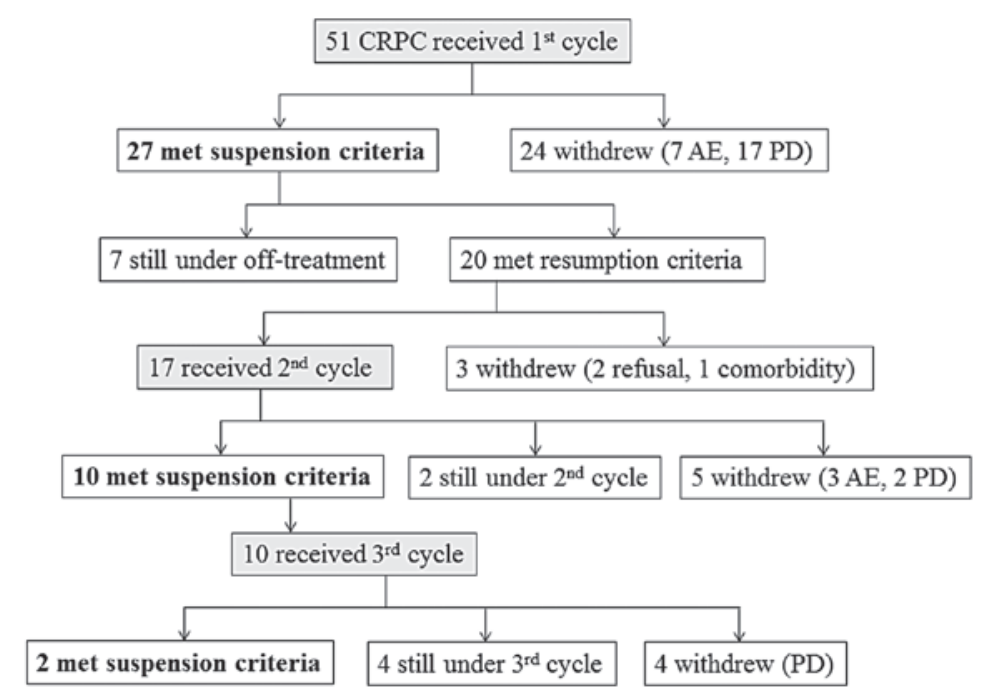

Figure 1. Flow diagram of the treatment outcomes of the 51 cases with castration-resistant prostate cancer (CRPC). A total of 27 patients (52.9\%) met the suspension criteria after the first, $10(19.6 \%)$ after the second and 2 (3.9\%) after the third treatment cycle. AE, adverse events; PD, progressive disease.

4 weeks apart (9). Four weeks were required after the termination of antiandrogen treatment (6 weeks for bicalutamide) to exclude antiandrogen withdrawal syndrome.

Treatment regimen. The patients were administered $75 \mathrm{mg} / \mathrm{m}^{2}$ docetaxel intravenously over 1 hour every 3 weeks, with concomitant administration of $1.0-2.0 \mathrm{mg} /$ day oral dexamethasone. Dexamethasone was continued at the same dose throughout the course of the treatment (10). Chemotherapy was suspended when the serum PSA level decreased to $<4 \mathrm{ng} / \mathrm{ml}$, with a reduction rate of $>50 \%$ from the baseline. Treatment was resumed when serum PSA increased to $>2 \mathrm{ng} / \mathrm{ml}$, with an increase rate of $>50 \%$ from the nadir.

Adverse events. The adverse events were prospectively recorded according to the Common Terminology Criteria for Adverse Events (CTCAE) 3.0 every 3 weeks in the outpatient clinic. Laboratory tests included a complete blood count, alkaline phosphatase (ALP), lactate dehydrogenase and PSA levels.

Statistical analysis. The Chi square test or the t-test were used for analysis of the patient characteristics. The overall survival was calculated from the initiation of docetaxel therapy. The log-rank test and the logistic regression model were used for univariate and multivariate analyses, respectively. P-values $<0.05$ were considered to indicate statistically significant differences. All the analyses were performed using the StatMate III software, version 3.07 (ATMS Co., Ltd., Tokyo, Japan).

\section{Results}

Patients and course of treatment. A total of 51 patients were enrolled in this study and followed up up to January, 2014, with a median observation period of 19.9 months (range, 0.7-48.6 months). Of the 51 patients, 27 (52.9\%) qualified for treatment suspension. Of those 27 patients who received intermittent therapy (intermittent cases) 7 remained under off-treatment at the end of study and the remaining 20 met the resumption criteria. Among the latter 20 subjects, 3 did not receive docetaxel therapy due to patients' refusal or respiratory comorbidity, whereas the remaining 17 patients received the second course of docetaxel therapy. After the second course, 5 patients withdrew from the regimen due to disease progression or adverse events, 2 continued with the chemotherapy and 10 entered the second off-treatment period. All 10 patients who met the suspension criteria twice received the third course of treatment and eventually met suspension criteria for the third time $(n=2)$, continued with the third therapy cycle $(n=4)$ or withdrew due to disease progression $(n=4)$ (Fig. 1). In total, 17 patients underwent one additional course (two courses in total) of docetaxel chemotherapy and 10 patients received two additional courses (three courses in total). The median duration of the off-treatment interval was 266 days for the first, 129.5 days for the second and 146.5 days for the third suspension period.

Characteristics of intermittent and non-intermittent cases. Intermittent cases tended to be younger at diagnosis (median age, 67.8 vs. 69.4 years, $\mathrm{P}=0.058$ ) and less likely to be diagnosed as Gleason score 8-10 (51.9 vs. $79.2 \%, \mathrm{P}=0.081$ ) compared to patients who did not meet the suspension criteria (non-intermittent cases) (Table I). During prior androgen-deprivation therapy, intermittent cases had been less frequently exposed to steroids (22.2 vs. $50.0 \%, \mathrm{P}=0.038$ ) or estrogen (14.8 vs. $41.7 \%$, $\mathrm{P}=0.032$ ) and attained a lower nadir of serum PSA (median 0.30 vs. $0.82 \mathrm{ng} / \mathrm{ml}, \mathrm{P}=0.029)$. Upon chemotherapy, the serum PSA level decreased to a significantly lower nadir in intermittent cases (median 8.37 vs. $107.6 \mathrm{ng} / \mathrm{ml}, \mathrm{P}=0.0004$ ). The dose of docetaxel and relative dose intensity tended to be higher in the intermittent cases $\left(72.0\right.$ vs. $69.3 \mathrm{mg} / \mathrm{m}^{2}, \mathrm{P}=0.087$ and 91.5 vs. $84.0 \%, \mathrm{P}=0.064$, respectively).

The multivariate analysis incorporating possible variables indicated low baseline PSA level (<median, $30.55 \mathrm{ng} / \mathrm{ml}$; odds ratio $=16.95, \mathrm{P}=0.010)$ and low Gleason score at diagnosis $(\leq 7$; odds ratio $=8.621, \mathrm{P}=0.040$ ) as significant factors for receiving intermittent therapy (Table II).

Adverse events. During the off-treatment period, leukopenia, thrombopenia, appetite loss, diarrhea, alopecia, nail changes 
Table I. Characteristics of patients $(n=51)$ with castration-resistant prostate cancer (CRPC).

\begin{tabular}{|c|c|c|c|}
\hline Characteristics & $\begin{array}{l}\text { Intermittent cases } \\
\qquad(\mathrm{n}=27)\end{array}$ & $\begin{array}{l}\text { Non-intermittent cases } \\
\qquad(\mathrm{n}=24)\end{array}$ & P-value \\
\hline \multicolumn{4}{|l|}{ Prior to docetaxel therapy } \\
\hline Initial PSA (ng/ml) & $57.0(5.80-2,750)$ & $217.2(14.1-5,407)$ & 0.422 \\
\hline Age at diagnosis (years) & $67.8(54.4-77.7)$ & $69.4(53.6-82.0)$ & 0.058 \\
\hline Gleason score 8-10 & $14(51.9)$ & $19(79.2)$ & 0.081 \\
\hline Radical prostatectomy or radiotherapy & 7 (25.9) & $5(20.8)$ & 0.923 \\
\hline PSA at the start of ADT & $42.2(1.70-2,750)$ & $217.8(0.88-5,407)$ & 0.477 \\
\hline Estramustine use & $11(40.7)$ & $13(54.2)$ & 0.338 \\
\hline Estrogen use & $4(14.8)$ & $10(41.7)$ & 0.032 \\
\hline Steroid use & $6(22.2)$ & $12(50.0)$ & 0.038 \\
\hline Nadir PSA (ng/ml) during ADT & $0.30(0.00-9.65)$ & $0.82(0.00-16.7)$ & 0.029 \\
\hline PSA response to prior ADT (\%) & $99.7(79.1-100.0)$ & $99.1(88.4-100.0)$ & 0.933 \\
\hline Time from diagnosis to CRPC status (months) & $18.2(0.53-163.4)$ & $24.2(5.47-106.3)$ & 0.408 \\
\hline PSA doubling time (months) & $1.88(0.23-16.3)$ & $2.67(0.72-22.1)$ & 0.560 \\
\hline PSA velocity (ng/ml/months) & $1.38(0.00-315.5)$ & $16.6(0.00-234.8)$ & 0.112 \\
\hline Time from diagnosis to docetaxel therapy (months) & $50.4(7.7-184.7)$ & $37.5(15.3-111.4)$ & 0.190 \\
\hline \multicolumn{4}{|l|}{ Upon docetaxel therapy } \\
\hline Age (years) & $72.0(55.9-82.5)$ & $73.9(59.5-87.0)$ & 0.281 \\
\hline Karnofsky performance status $\geq 80 \%$ & $26(96.3)$ & $23(95.8)$ & 0.524 \\
\hline PSA (ng/ml) & $8.37(1.80-173.6)$ & $107.6(8.21-794.6)$ & 0.0004 \\
\hline Lactate dehydrogenase (IU/l) & $219(116-907)$ & $266.5(166-1,200)$ & 0.090 \\
\hline Alkaline phosphatase (IU/l) & $253(46-4,348)$ & $227(115-3,462)$ & 0.905 \\
\hline Hemoglobin (g/dl) & $12.1(9.4-14.4)$ & $11.2(8.1-14.2)$ & 0.142 \\
\hline Bone metastasis & $21(77.8)$ & $20(83.3)$ & 0.884 \\
\hline Lymph node metastasis & $17(63.0)$ & $15(62.5)$ & 0.973 \\
\hline Analgesics use & $5(18.5)$ & $9(37.5)$ & 0.669 \\
\hline \multicolumn{4}{|l|}{ Docetaxel therapy } \\
\hline Dose $\left(\mathrm{mg} / \mathrm{m}^{2}\right)$ & $72.0(52.3-77.8)$ & $69.3(26.2-74.9)$ & 0.087 \\
\hline Relative dose intensity (\%) & $91.5(62.8-104.7)$ & $84.0(32.8-101.8)$ & 0.064 \\
\hline
\end{tabular}

Values are presented as median (range) or as no. (\%). PSA, prostate-specific antigen; ADT, androgen-deprivation therapy.

Table II. Multivariate analysis of significant factors for chemotherapy suspension.

\begin{tabular}{|c|c|c|}
\hline Factors & Odds ratio $(95 \% \mathrm{CI})$ & P-value \\
\hline Age $<68.5^{\text {a }}$ years at diagnosis & $4.367(0.690-27.78)$ & 0.117 \\
\hline Gleason score $\leq 7$ & $8.621(1.105-66.67)$ & 0.040 \\
\hline History of steroid use & $0.291(0.044-1.928)$ & 0.201 \\
\hline History of estrogen use & $0.429(0.067-2.741)$ & 0.371 \\
\hline PSA nadir $<0.52 \mathrm{ng} / \mathrm{ml}^{\mathrm{a}}$ during ADT & $1.047(0.192-5.714)$ & 0.957 \\
\hline PSA $<30.55 \mathrm{ng} / \mathrm{ml}^{\mathrm{a}}$ at the start of docetaxel & $16.95(1.953-142.9)$ & 0.010 \\
\hline $\mathrm{LDH}<251 \mathrm{IU} / \mathrm{ml}^{\mathrm{a}}$ at the start of docetaxel & $2.584(0.425-15.63)$ & 0.302 \\
\hline Relative dose intensity $<89.6 \%^{\mathrm{a}}$ & $0.274(0.026-2.888)$ & 0.281 \\
\hline
\end{tabular}

${ }^{\mathrm{a}}$ Median values. CI, confidence interval; PSA, prostate-specific antigen; ADT, androgen-deprivation therapy; LDH, lactate dehydrogenase.

and fatigue persisted in none $(0.0 \%), 2(7.4 \%), 1(3.7 \%)$, $1(3.7 \%), 3(11.1 \%), 2(7.4 \%)$ and $3(11.1 \%)$ of the 27 intermittent cases, respectively (Table III). However, sensory and/or motor neuropathy persisted in 12 of the 27 cases $(44.4 \%)$. There were no observed grade $3 / 4$ adverse events persisting during the chemotherapy-free period. 
Table III. Adverse events.

\begin{tabular}{lccc}
\hline & \multicolumn{2}{c}{ On-treatment period } & Off-treatment period \\
\cline { 2 - 3 } Adverse events & $24(88.9)$ & Grades 3, 4, no. (\%) & Persisting AEs, no. (\%) \\
\hline Leukopenia & $6(22.2)$ & $14(51.9)$ & $0(0.0)$ \\
Thrombopenia & $6(22.2)$ & $0(0.0)$ & $2(7.4)$ \\
Appetite loss & $4(14.8)$ & $0(0.0)$ & $1(3.7)$ \\
Diarrhea & $14(51.9)$ & $0(0.0)$ & $1(3.7)$ \\
Alopecia & $11(40.7)$ & $0(0.0)$ & $3(11.1)$ \\
Nail changes & $6(22.2)$ & $0(0.0)$ & $2(7.4)$ \\
Fatigue & $14(51.9)$ & $1(3.7)$ & $3(11.1)$ \\
Neuropathy (sensory, motor) & & $0(0.0)$ & $12(44.4)$ \\
\hline
\end{tabular}

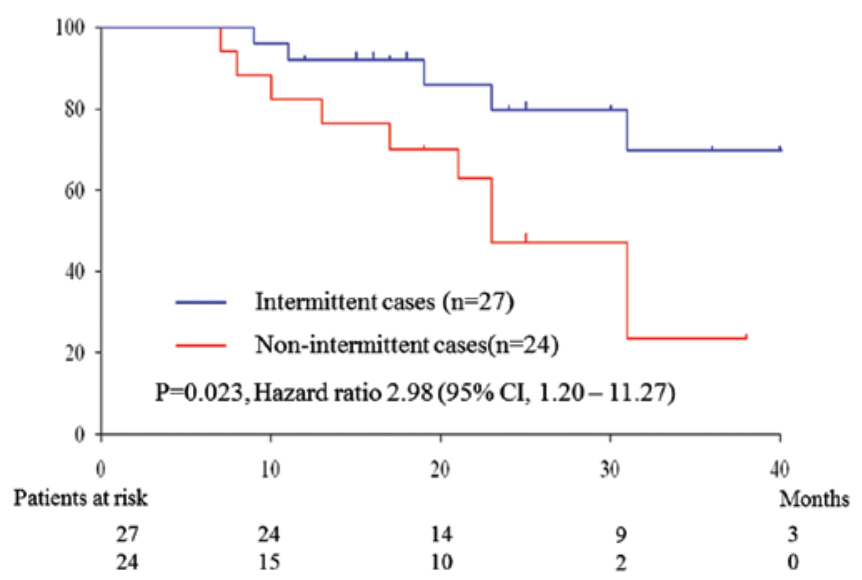

Figure 2. Overall survival from the start of docetaxel chemotherapy. The survival was better for intermittent cases (hazard ratio $=2.98,95 \%$ confidence interval: $1.20-11.27, \mathrm{P}=0.023$ ).

Survival. During a median follow-up period of 19.9 months, 15 patients succumbed to prostate cancer ( 6 intermittent and 9 non-intermittent cases). The overall survival was significantly longer in the intermittent cases by the log-rank test (hazard ratio $=2.98, \mathrm{P}=0.023$ ) (Fig. 2).

\section{Discussion}

The present study prospectively investigated the feasibility of a protocol of intermittent docetaxel chemotherapy for CRPC. Chemotherapy was suspended when serum PSA level decreased to $<4 \mathrm{ng} / \mathrm{ml}$ and the reduction rate was $>50 \%$. The suspension was maintained as long as serum PSA level remained $<2 \mathrm{ng} / \mathrm{ml}$ or the increase rate from the nadir was $<50 \%$. Once the suspension criteria were no longer fulfilled, docetaxel chemotherapy was resumed. The protocol was applicable to 27 of the 51 CRPC patients (52.9\%), with a median off-treatment period of 266 days. During the drug holidays, the majority of the adverse events, apart from neuralgia, subsided. The overall survival was significantly longer among patients who met the suspension criteria.

Previous studies indicated that intermittent docetaxel chemotherapy was feasible in approximately half of the cases, although the patients' backgrounds and the criteria determining docetaxel suspension and resumption differed from the ones in our study (6-8). Although the planned dose intensities were similar among the studies ( $25 \mathrm{mg} / \mathrm{m}^{2} /$ week), the serum PSA level at the initiation of docetaxel therapy was higher in the study of Beers et al (median, $104.65 \mathrm{ng} / \mathrm{ml}$ ) (7), which may explain the lower treatment suspension rate (16.0\%) observed in their study.

Our multivariate analysis identified lower baseline PSA level as a significant factor for receiving intermittent therapy. Beer et al (7) also reported that intermittent cases had a lower PSA level, a better performance status, a higher hemoglobin concentration and a lower serum ALP level. These results suggest that cases with less advanced disease may achieve a better response to docetaxel therapy and, thus, have a higher chance to undergo intermittent chemotherapy.

As regards the quality of life (QOL) during the off-treatment period, Mountzios et al analyzed 35 cases, using a questionnaire covering several aspects of QOL assessment and found that all three domains of QOL assessment (general condition, daily activities and symptom-oriented evaluation) improved during the chemotherapy-free interval, concluding that the intermittent docetaxel therapy was a clinically viable therapeutic strategy (8). The authors of that study also reported that sensory neuropathy occurred in $18 \%$ of their cases, but only mentioned that all treatment-related toxicities subsided during the chemotherapy-free period (8).

Our study prospectively monitored adverse events using CTCAE and confirmed that all the adverse events, apart from motor and sensory neuropathy, fully subsided in almost all the cases. When reviewing studies on docetaxel therapy for breast cancer, Alken et al concluded that peripheral neuropathy may only be managed with appropriate dose interruptions and scheduling (11).

Our study was performed in a single institution and our sample number was limited. Further prospective studies of a larger size or with comparative arms are required to determine the clinical feasibility and significance of the intermittent therapy for CRPC patients.

Our intermittent regimen of docetaxel chemotherapy was found to be feasible for CRPC, since it may reduce adverse events without compromising the oncological outcome. 


\section{References}

1. http://www.nccn.org/professionals/physician_gls/f_guidelines. asp. Access date, $20^{\text {th }}$ November, 2014

2. http://www.uroweb.org/guidelines/. Access date, $20^{\text {th }}$ November, 2014

3. Tannock IF, de Wit R, Berry WR, Horti J, Pluzanska A, Chi KN, Oudard S, Théodore C, James ND, Turesson I, Rosenthal MA and Eisenberger MA; TAX 327 Investigators: Docetaxel plus prednisone or mitoxantrone plus prednisone for advanced prostate cancer. N Engl J Med 351: 1502-1512, 2004.

4. Petrylak DP, Tangen CM, Hussain MH, Lara PN Jr, Jones JA, Taplin ME, Burch PA, Berry D, Moinpour C, Kohli M, Benson MC, Small EJ, Raghavan D and Crawford ED: Docetaxel and estramustine compared with mitoxantrone and prednisone for advanced refractory prostate cancer. N Engl J Med 351: 1513-1520, 2004.

5. Naito S, Tsukamoto T, Koga H, Harabayashi T, Sumiyoshi Y, Hoshi S and Akaza H: Docetaxel plus prednisolone for the treatment of metastatic hormone-refractory prostate cancer: a multicenter phase II trial in Japan. Jpn J Clin Oncol 38: 365-372, 2008.

6. Soga N, Kato M, Nishikawa K, Hasegawa Y, Yamada Y, Kise H, Arima K and Sugimura Y: Intermittent docetaxel therapy with estramustine for hormone-refractory prostate cancer in Japanese patients. Int J Clin Oncol 14: 130-135, 2009.
7. Beer TM, Ryan CW, Venner PM, Petrylak DP, Chatta GS, Ruether JD, Chi KN, Young J and Henner WD; ASCENT (AIPC Study of Calcitriol ENhancing Taxotere) Investigators: Intermittent chemotherapy in patients with metastatic androgen-independent prostate cancer: results from ASCENT, a double-blinded, randomized comparison of high-dose calcitriol plus docetaxel with placebo plus docetaxel. Cancer 112: 326-330, 2008.

8. Mountzios I, Bournakis E, Efstathiou E, Varkaris A, Wen S, Chrisofos M, Deliveliotis C, Alamanis C, Anastasiou I, Constantinides C, Karadimou A, Tsiatas M, Papadimitriou C, Bamias A and Dimopoulos MA: Intermittent docetaxel chemotherapy in patients with castrate-resistant prostate cancer. Urology 77: 682-687, 2011.

9. The Japanese Urological Association, the Japanese Society of Pathology and Japan Radiological Society: General rule for Clinical and Pathological Studies on Prostate Cancer. 4th edition. Kanehara \& Co., Ltd., Tokyo, 2010.

10. Kume H, Suzuki M, Fujimura T, Fukuhara H, Enomoto Y, Nishimatsu H, Ishikawa A and Homma Y: Docetaxel as a vital option for corticosteroid-refractory prostate cancer. Int Urol Nephrol 43: 1081-1087, 2011.

11. Alken S and Kelly CM: Benefit risk assessment and update on the use of docetaxel in the management of breast cancer. Cancer Manag Res 5: 357-365, 2013. 